\title{
Evaluation of Factors Affecting Employees' Performance Using Artificial Neural Networks Algorithm: The Case Study of Fajr Jam
}

\author{
Mohammad Rahmanidoust ${ }^{1}$, Jianguo Zheng ${ }^{1}$ \\ ${ }^{1}$ Glorious Sun School of Business and Management, Donghua University, Shanghai 200051, China \\ Correspondence: Mohammad Rahmanidoust, Glorious Sun School of Business and Management, Donghua \\ University, Shanghai 200051, China.
}

Received: June 24, 2019

doi:10.5539/ibr.v12n10p86

\author{
Accepted: September 9, $2019 \quad$ Online Published: September 26, 2019 \\ URL: https://doi.org/10.5539/ibr.v12n10p86
}

\begin{abstract}
Human resources are the most valuable assets of any organization. Therefore, human resources performance has the greatest impact on the organization's performance and its ability to operate. Many factors affect the performance of employees in organizations. In this research, we seek to evaluate the factors affecting the performance of Fajr Jam refinery employees. For this purpose, firstly, the literature of the research, the indicators affecting the performance of employees were identified and the conceptual model of the problem was formed. Then, the required data were collected using a standard questionnaire based on the conceptual model of the problem among employees of FJG Company. After assessing the validity and reliability of the collected data, it is time to evaluate the performance of the indicators. For this purpose, an artificial neural network algorithm was used to estimate the efficiency boundary values. After calculating the efficiency values in the presence of all the indices, each indices were eliminated from the conceptual model and again the efficiency values were estimated. Now, by comparing the performance statistics in the state before and after the removal of each indicator from the conceptual model, the degree and the mode of its effect are determined. The results of this study indicate that the "payroll" indicators, "environmental conditions" and "reporting culture" are the strengths of the system under review, and are now at an appropriate level. Also, the results indicate a negative impact on the indicators of "awareness", "system planning and preparation for critical situations," "amenities," "training," and "job security" in the system under review.
\end{abstract}

Keywords: performance evaluation, efficiency boundary analysis, artificial neural networks, statistical methods

\section{Introduction}

Human resources are one of the most important sources of each organization and system. Human resource performance in a system has a direct and significant impact on overall system performance. Proper performance and employee effectiveness is one of the key keys to the success of organizations. That is why the successful world organizations are working hard to identify and manage the factors affecting employee behavior and behavior. Obviously, with this approach, performance management as a tool for improving performance and promoting employee behavior is of great importance in the management of organizations (Azadeh et al., 2017). Therefore, evaluation of human resources performance has always been one of the most important issues for managers of organizations. Human resources performance evaluation can be done from different aspects and directions. To this end, first, the factors affecting the performance of employees in organizations should be identified and then, using qualitative or quantitative methods, they should be evaluated. In this way, one can examine the impact of each of the influential factors and identify the weaknesses and strengths of the organization in dealing with human resources. It should be noted that various factors affect the performance of employees in an organization (Kennerley and Neely, 2002). Among the factors influencing the performance of the employees, one can refer to the work environment, the set of factors related to job satisfaction, the ability of the information system, the teamwork spirit, the level of knowledge and skills, the capabilities of the tools, the support and commitment of senior management organization, the proportion between education and post Organizational and so on.The researchers have presented a variety of approaches and approaches to identify and evaluate the factors affecting employee performance. One of these methods can be referred to methods based on multi-criteria decision making techniques, artificial intelligence, conceptual models and statistical methods. The purpose of this study is to evaluate the factors affecting the performance of the employees of FJJ Company 
Refinery Company using the algorithm based on artificial neural networks. To this end, the effective factors were first identified and then, using artificial neural network algorithm, we identify the weaknesses and strengths of the unit. The results of this research can help the unit management to improve the performance of employees.

The issue of the importance and necessity of human resources management has begun in the Western countries from the early 1980s, and has involved many researchers in the issue of human resource performance assessment and in the further management of human resources. Initially, researchers sought conceptual assessments using a variety of approaches such as applied psychology and humanities (Meyer et al., 1989). Huselid et al. (1997) evaluated 293 small and medium enterprises in the United States and used questionnaires, face to face interviews and non-personal interviews to identify factors affecting employee performance and the impact of these factors. Their research suggests that the effectiveness of employee performance on the organization's cash flows, equity and market value, and the productivity of the organization. By highlighting the importance of the issue in the performance of the organization in the 1990s, many researchers entered the field of identifying and evaluating factors affecting the performance of the staff of an organization. Jiang et al. (2012) evaluated the relationship between the way human resource management in an organization and employee performance. To this end, researchers have assessed the different parts of the HRM system and identified their relationship with the performance of the staff. Hoque (2013) assessed the human resources management system from the strategic level to the operational level and its impact on the performance of staff in the hotel industry. Shields et al. (2015) in his book evaluates the payroll system and rewards the performance of human resources in organizations. Hadden et al. (2007) introduced a booklet for training, management and human resource performance assessment, in which all known and well-known methods were introduced in a practical manner. Researchers have used many techniques to evaluate factors and criteria in research. A great deal of research in this field is made up of multi-criteria decision-making techniques. One of these techniques, which is well accepted by the researchers, is data envelopment analysis. Data Envelopment Analysis is a tool based on linear and capability planning that is used today in most countries of the world to evaluate system performance with multiple inputs and multiple outputs. Data Envelopment Analysis represents a nonparametric technique for measuring and evaluating the relative performance of a set of organizations with definitive inputs and outputs. The reason for the wider acceptance of the method of data envelopment analysis is the possibility of examining complex and often uncertain relationships between multiple inputs and multiple outputs. In addition, DEA is a non-parametric method used by linear programming to calculate productivity in a given set of decision-making units (Heidari et al., 2017). Since the DEA is a technique for assessing the relative efficiency of the decision-making units, at least one unit is located on the boundary and the rest of the units are below it. So far, researchers have used many data envelopment analysis techniques to assess the factors and their impact on different systems of production and industry (Thanassoulis, 1993; Thanassoulis et al., 1996; Liu et al., 2000; Wang et al., 2009; Luna et al., 2013). In addition to using data envelopment analysis, some researchers have developed intelligence-based intelligence algorithms based on data envelopment analysis that has been highly applied and so far a lot of research has been done on them. Azadeh et al. (2015b) evaluated the factors affecting the operation of the gas refinery using an algorithm based on data envelopment analysis. In this study, in addition to financial factors and factors, operational factors were also considered in the unit performance evaluation. In addition to data envelopment analysis, researchers in this study also used key component analysis techniques and finally compared the results of each approach. The results of this research have shown that the data-based approach to data envelopment analysis is more robust and more effective than data disturbances. Azadeh et al. (2015a) investigated the factors affecting the performance of electricity distribution units in Iran using a data-based data analysis algorithm. One of the advantages of this algorithm is to determine the current status of the factors and factors in the case being studied, which means that this approach is a case-centric approach, which makes this approach more efficient and capable. Faed et al. (2015) used this technique to assess the strengths and weaknesses of a customer service system. In order to calculate the performance index in the performance evaluation algorithm, artificial neural networks can be used in addition to data envelopment analysis. So far, many researchers have used artificial intelligence based artificial intelligence algorithms to evaluate performance and factors in systems. The superiority of this approach to data envelopment analysis is the ability to consider non-linear relationships between data (data envelopment analysis is a linear model and considers only linear relationships.) Also, a neural network-based algorithm can be a useful boundary. This is an accidental flaw in the data envelopment analysis, which increases the accuracy and performance of the algorithm. Azadeh et al. (2013b) in an experiment using an artificial neural network algorithm, evaluation and optimization of individual characteristics along with health, safety, environment and ergonomics factors. Azadeh et al. (2013a)developed an artificial neural network based intelligent algorithm evaluated the factors affecting the workload, health, safety and environmental and ergonomics in a gas dilution unit. 
Human resources are one of the most important sources of each organization and system. Human resource performance in a system has a direct and significant impact on overall system performance. Proper performance and employee effectiveness is one of the key keys to the success of organizations. That is why the successful world organizations are working hard to identify and manage the factors affecting employee behavior and behavior. Obviously, with this approach, performance management as a tool for improving performance and promoting employee behavior is of great importance in the management process of organizations. So far, no research has considered all safety, job satisfaction, and work environment factors integrated to evaluate employee performance at Fajr Refinery. In this regard, the current research is unique and can provide practical solutions to improve the performance of the employees of the unit. Also, an artificial neural network based analytical technique is new and has not been used in the evaluation of the integrated performance of the refinery staff. The purpose of the present study is to evaluate the performance of Fajr refinery staff and identify the strengths and weaknesses in their productivity and performance. By evaluating these, we can propose ways to improve the performance of the employees, which will result in improving the performance of the industrial unit under investigation. All industrial units similar to Fajr Jam GHP and petrochemical units can use the current research approach to evaluate the performance of their employees. Also, the results of sensitivity analysis obtained for Fajr refinery can be generally Method of conducting research. The purpose of this study was to identify and evaluate the factors affecting the employees of Fajr Jam Gas Refining Company. For this purpose, using library studies and authoritative scientific references, the factors influencing the performance of the staff have been identified and identified and designed to achieve the objectives of the problem of a conceptual model. The structure of the conceptual model is considered and the factors affecting employee performance are presented below:

- Salary: It should be noted that one of the main reasons for people's tendency to address material needs. The salary paid to employees has always been one of the most important indicators affecting the satisfaction of employees, both in industrial environments and in service environments. So far, much research has been done on payroll systems to increase employee satisfaction.

- Environmental conditions: Another important factor affecting the performance of employees in industrial environments is the environmental conditions of the workplace. Among the environmental factors that can be mentioned are temperature, humidity, noise, air quality and other factors. In general, the environmental condition indicator has been designed and defined as a comprehensive indicator covering all factors and indicating the perception and overall satisfaction of the individual's working conditions.

- Senior Management Support: Many studies have so far been conducted on the role and influence of each organization's senior management on the organization's performance. In addition to the strategic role of each organization's top management in planning and promoting the organization, senior management has a major role in human resource management and their performance and satisfaction. This indicator, which is one of the accepted indicators in preventive safety topics such as reversible engineering (resilience), can affect employee satisfaction and performance in critical industrial units.

- Awareness: In the early twentieth century, following many disasters and catastrophes in industrial units, many researchers focused on identifying and evaluating the role of human resources in system failure and error. One of the most important and influential indicators finally introduced in the framework of reverse engineering as an approach to preventive safety. Awareness is the level of awareness of the employees of each system of accidents and safety risks present in the system. It should be noted that many companies and industries are trying to keep track of incidents in the system even hidden. Researchers have shown that awareness of emerging accidents and workplace safety tips can greatly affect employee performance and help them prevent future accidents.

- Reporting: The culture of reporting is the amount of comfort and ease of human resources in reporting cases and safety issues and work hazards.

- Spirituality of teamwork and collaboration: Another known and influential factor in employee performance is the team spirit and collaboration with other employees at the workplace.

- System planning and preparation for critical situations: One of the issues affecting system performance and system planning staff is the critical and so-called risk and safety planning.

- Facilities: Company amenities such as catering, accommodation and nutrition are also factors affecting the performance of employees in industrial environments. 
- Training: One of the most important factors affecting the performance of employees in sensitive industrial units such as petrochemicals and refinery staff before entering the work environment and preparing them for performing assigned tasks.

- Job security is one of the most important factors affecting the satisfaction of employees and, as a result, their job security. The sense of job security creates job satisfaction and commitment in the staff and can significantly increase their performance and efficiency.

\section{Methodology}

\subsection{Data Collection}

After identifying the effective factors and designing the conceptual model, the problem is the time to collect data. For this purpose, a standard questionnaire based on a 10-point scale is designed based on a conceptual model. A questionnaire designed for current research is presented in the table in Appendix.

Given that the required data are prepared using a questionnaire, we need a tool to examine the validity and reliability of the data. To test the validity and reliability of the data in this study, the test method is used. The methodology is that at first, two random samples are collected from the data collected from the questionnaires for one question. Then, using the $t$ test, the mean of these two samples is compared, if at the level of 0.05 this test it does not exclude the fact that society is one-sided and the data is trusted and accepted. If this test is rejected, then you are looking for distorted values, identify and remove them, and then retest the average.

After distributing the designed questionnaires randomly, an intelligent algorithm based on artificial neural networks turns to the research community and collecting the required data. Considering the purpose of this study is to evaluate the factors affecting the performance of the employees of FJJ Company, the artificial neural network algorithm is used in this research, which is very flexible, powerful and new in this field. It also has the ability to consider non-linear relationships as opposed to data-based data analysis models.

\subsection{Artificial Neural Network Algorithm}

This algorithm uses artificial neural networks (ANN) and statistical methods such as mean test and correlation coefficient to evaluate the factors and performance of the systems. The steps to implement this algorithm are as follows:

1. Determine the optimal structure of artificial neural network according to the model and data available in the problem

After collecting the questionnaire and extracting the required data, according to the conceptual model, the data input and output data are separated and categorized. Then, we introduce and codify the various models of artificial neural networks including the ANN-MLP and the radial base function (RBF).

After implementing different models of neural networks in MATLAB software, it is time to examine the different structures of these models to identify the optimal structure based on the data and the model of the present research. This process is referred to as parameter setting. After designing different structures of the neural networks for each model, taking into account the data collected in the problem, each structure in the MATLAB software is adjusted and implemented using the encoded code and the corresponding absolute error (MAE) to any structure. At the end of the structure, the absolute error value is chosen as the optimal structure. This process is performed for the model and ultimately the optimal structure of the neural network is determined for the model.

2. After determining the optimal structure of the artificial neural network for the model, using the data collected and based on the identified conceptual model including inputs and outputs, a neural network with an optimal structure is implemented and the outputs of the model are predicted. . At this point, $15 \%$ of the data is used to train the neural network and $15 \%$ to test the network. At the end of this step, the output values of the model are predicted to be related to the optimal structure and the corresponding data. This value is PBest.

3. At this stage, we seek to calculate the efficiency index for the complete state (presence of all factors and model factors). To do this, the following steps should be followed. First we call the actual output values of the questionnaire for PReal (i).

4. We calculate the error value using the following formula (1). 


$$
E_{\text {ir }}=O_{\text {real }(i r)}-O_{A N N(i r)}(r=1, \ldots, R \text { and } i=1, \ldots, I)
$$

5. Calculate relative error values using the following formula (2)

$$
E_{\text {ir }}^{\prime}=\left(E_{\text {ir }} / O_{\text {ANN(ir) }}\right) \quad(r=1, \ldots, R \text { and } i=1, \ldots, I)
$$

6. Now we identify and call the highest relative error value for each factor in the model (3)

$$
E_{k}=\max \left(E_{r}^{\prime}\right)
$$

7. We calculate the shifts for each DMU using the following formula (4)

$$
S h_{i r}=E_{k} * \frac{O_{A N N(i r)}}{O_{A N N(i k)}} \quad(r=1, \ldots, R \text { and } i=1, \ldots, I)
$$

In the above formula, the amount of expected output is calculated using the neural network for the decision unit, which has the highest relative error value.

8. In the final stage, the efficiency of each decision unit is obtained using the following formulas(5-6).

$$
\begin{gathered}
F_{i r}=P_{i r} /\left(O_{A N N(i r)}+S h_{i r}\right) \\
\bar{F}_{i}=\frac{\sum_{r=1}^{R} F_{i r}}{J}
\end{gathered}
$$

9. To determine the impact of each factor on the model, we remove each of the factors and repeat steps 4 through 8 to calculate the efficiency of each mode.

10. Sensitivity analysis of the model based on calculated performance values.

At this stage, for the model, the obtained efficiency values are considered and then, using statistical methods such as t-test and Pearson correlation coefficient test, sensitivity analysis is performed and ultimately the effect level of each factor is determined. The general logic used in this step is to compare the performance values before and after the removal of each index from the conceptual model of the problem. If, after eliminating performance values, the average increase, it can be claimed that the deleted index has a negative effect, and vice versa.

\section{Results and Findings}

The symbols and agents guide are presented in Table 1.

Table 1. A guide to symbols and factors in the problem model

\begin{tabular}{cc}
\hline Index & Factor \\
\hline O1 & Salary and wage \\
O2 & Work environment \\
O3 & Top management support \\
O4 & Awareness \\
O5 & Reporting culture \\
O6 & Teamwork \\
O7 & Preparedness \\
O8 & Facility and welfare \\
O9 & Training \\
O10 & Job security \\
\hline
\end{tabular}

Now, as described in the previous section, first of all, the reliability and reliability of the data collected for research should be ensured. For this purpose, Cronbach's alpha test is used to assess the validity of the data and 
the mean $t$ test is used to evaluate the reliability of the data. The results of this step are presented in Table 2.

Table 2. Results of validity and reliability of collected data Cronbach's alpha

\begin{tabular}{cccc}
\hline Factor & Alpha Cronbach & p-value & Mean \\
\hline Salary and wage & 0.612 & 0.142 & 2.5744 \\
Work environment & 0.731 & 0.433 & 4.8014 \\
Top management support & 0.755 & 0.321 & 8.4326 \\
Awareness & 0.699 & 0.220 & 7.3758 \\
Reporting culture & 0.735 & 0.202 & 7.0567 \\
Teamwork & 0.678 & 0.311 & 3.7801 \\
Preparedness & 0.710 & 0.178 & 5.7234 \\
Facility and welfare & 0.724 & 0.089 & 5.3829 \\
Training & 0.747 & 0.561 & 6.4609 \\
Job security & 0.643 & 0.318 & 4.0567 \\
\hline
\end{tabular}

As described in before, this section first identifies an optimal neural network structure based on problem data and then uses the identified network to implement the algorithm steps based on artificial neural networks. To this stage in the application of artificial neural networks, the parameter setting stage is also called.

In this study, we consider two models of artificial neural networks MLP and RBF that have been accepted as well-known as artificial modular neural networks. In order to evaluate and identify the different structures of these two models, we have identified potentially and potentially powerful scenarios based on authoritative articles and scientific references. The different structures identified in Tables 3 and 4 are presented. In this way, parameter setting is performed randomly, also called try and error.

Table 3. Various Artificial Neural Network Models of the ANN-MLP Model

\begin{tabular}{|c|c|c|c|c|c|c|c|}
\hline $\begin{array}{c}\text { ANN } \\
\text { number }\end{array}$ & $\begin{array}{l}\text { Training } \\
\text { function }\end{array}$ & $\begin{array}{l}\text { Number } \\
\text { of HLs }\end{array}$ & $\begin{array}{l}\text { TF of the } \\
\text { first HL }\end{array}$ & $\begin{array}{c}\text { Number of } \\
\text { neurons in first HL }\end{array}$ & $\begin{array}{l}\text { TF of the } \\
\text { second HL }\end{array}$ & $\begin{array}{l}\text { Number of neurons in } \\
\text { the second HL }\end{array}$ & $\begin{array}{l}\text { TF of } \\
\text { the OL }\end{array}$ \\
\hline 1 & LM & 1 & tansig & 5 & & & purelin \\
\hline 2 & GD & 2 & logsig & 4 & logsig & 4 & purelin \\
\hline 3 & BFGS & 1 & logsig & 6 & & & purelin \\
\hline 4 & LM & 2 & tansig & 5 & tansig & 5 & purelin \\
\hline 5 & GDA & 2 & logsig & 11 & tansig & 11 & purelin \\
\hline 6 & OSS & 1 & tansig & 35 & & & purelin \\
\hline 7 & LM & 1 & logsig & 30 & & & purelin \\
\hline 8 & BFGS & 2 & logsig & 8 & tansig & 8 & purelin \\
\hline 9 & GDA & 1 & logsig & 3 & & & purelin \\
\hline 10 & OSS & 2 & tansig & 17 & tansig & 17 & purelin \\
\hline 11 & LM & 2 & logsig & 15 & tansig & 15 & purelin \\
\hline 12 & BFGS & 1 & tansig & 40 & & & purelin \\
\hline 13 & GDA & 1 & tansig & 30 & & & purelin \\
\hline 14 & GDX & 2 & logsig & 7 & $\log \operatorname{sig}$ & 7 & purelin \\
\hline
\end{tabular}

Table 4. Various Artificial Neural Network Models of the RBF Model

\begin{tabular}{ccc}
\hline Number & Spread & Maximum number of neurons \\
\hline 1 & 2 & 25 \\
$\mathbf{2}$ & $\mathbf{4}$ & $\mathbf{2 0}$ \\
3 & 0.5 & 30 \\
4 & 2 & 15 \\
5 & 4 & 12 \\
6 & 3 & 20 \\
7 & 1 & 45 \\
\hline
\end{tabular}

After identifying the various structures of artificial neural networks, it is time to review and evaluate each of the structures using the problem data. Considering that in this research the concept model for evaluating the integrated performance of the indicators of job satisfaction, safety and industrial hygiene is under study, we use the data collected for indicators to evaluate the performance of each of the indicators. After implementing a variety of neural network structures, the mean absolute error values for each structure are obtained and presented in Tables 5 and 6. 
Table 5.The results of absolute error values related to each structure of the MLP neural

\begin{tabular}{|c|c|c|c|c|c|c|c|c|}
\hline $\begin{array}{c}\text { ANN } \\
\text { numbe } \\
\text { r }\end{array}$ & $\begin{array}{l}\text { Training } \\
\text { function }\end{array}$ & $\begin{array}{l}\text { Number } \\
\text { of HLs }\end{array}$ & $\begin{array}{l}\text { TF of the } \\
\text { first HL }\end{array}$ & $\begin{array}{c}\text { Number of } \\
\text { neurons in first } \\
\text { HL }\end{array}$ & $\begin{array}{l}\text { TF of the } \\
\text { second HL }\end{array}$ & $\begin{array}{l}\text { Number of neurons } \\
\text { in the second HL }\end{array}$ & $\begin{array}{l}\text { TF of } \\
\text { the OL }\end{array}$ & $\begin{array}{l}\text { Mean } \\
\text { Absolute } \\
\text { Error }\end{array}$ \\
\hline 1 & LM & 1 & $\operatorname{tansig}$ & 5 & & & purelin & 0.2531 \\
\hline 2 & GD & 2 & logsig & 4 & logsig & 4 & purelin & 0.2132 \\
\hline 3 & BFGS & 1 & logsig & 6 & & & purelin & 0.2347 \\
\hline 4 & $\mathrm{LM}$ & 2 & tansig & 5 & tansig & 5 & purelin & 0.1867 \\
\hline 5 & GDA & 2 & logsig & 11 & tansig & 11 & $\begin{array}{c}\text { pureli } \\
\text { n }\end{array}$ & 0.1673 \\
\hline 6 & OSS & 1 & tansig & 35 & & & purelin & 0.2237 \\
\hline 7 & LM & 1 & logsig & 30 & & & purelin & 0.2188 \\
\hline 8 & BFGS & 2 & logsig & 8 & tansig & 8 & purelin & 0.1908 \\
\hline 9 & GDA & 1 & $\log \operatorname{sig}$ & 3 & & & purelin & 0.3132 \\
\hline 10 & OSS & 2 & tansig & 17 & tansig & 17 & purelin & 0.1653 \\
\hline 11 & LM & 2 & logsig & 15 & tansig & 15 & purelin & 0.1590 \\
\hline 12 & BFGS & 1 & tansig & 40 & & & purelin & 0.2852 \\
\hline 13 & GDA & 1 & tansig & 30 & & & purelin & 0.2556 \\
\hline 14 & GDX & 2 & $\log \operatorname{sig}$ & 7 & $\log \operatorname{sig}$ & 7 & purelin & 0.1983 \\
\hline
\end{tabular}

Table 6. The results of absolute error values for each structure of the RBF neural network model

\begin{tabular}{cccc}
\hline Number & Spread & Maximum number of neurons & Mean Absolute Error \\
\hline 1 & 2 & 25 & 0.1586 \\
$\mathbf{2}$ & $\mathbf{4}$ & $\mathbf{2 0}$ & $\mathbf{0 . 1 4 8 9}$ \\
3 & 0.5 & 30 & 0.2596 \\
4 & 2 & 15 & 0.1762 \\
5 & 4 & 12 & 0.1877 \\
6 & 3 & 20 & 0.1701 \\
7 & 1 & 45 & 0.3214 \\
\hline
\end{tabular}

As shown in Tables 5 and 6, optimal neural network structures for MLP and RBF models are identified for both issues. Finally, based on the mean value of absolute error, the optimal structure of the present study, which is used in the next steps, is presented in Table 7.

Table 7. Optimal neural network structures for the conceptual model of the problem

\begin{tabular}{cl}
\hline ANN model & MAPE \\
\hline ANN-RBF, Max Neurons $=20$, Spread $=4$ & 0.1489 \\
\hline
\end{tabular}

Now, using the optimal identified structures, we proceed with the algorithm steps for the conceptual model of the problem to find the efficiency values.After identifying the optimal structure of the neural network for the problem data, it is time to calculate the efficiency values according to the steps outlined in methodology.By using the inputs and outputs identified and the standard values obtained in the previous section and the optimal structure of the identified neural network, we are training the neural network and predicting the output values. Then, by proceeding with the steps of the algorithm, we achieve performance values. The results of this section are presented in Table 8.

Table 8 . The efficiency values associated with the presence of all factors in the integrated model

\begin{tabular}{cccccccccc}
\hline DMU & Efficiency & DMU & Efficiency & DMU & Efficiency & DMU & Efficiency & DMU & Efficiency \\
\hline 1 & 0.139613 & 30 & 0.041683 & 59 & 0.190098 & 88 & 0.062308 & 117 & 0.062601 \\
2 & 0.157462 & 31 & 0.072023 & 60 & 0.092356 & 89 & 0.208625 & 118 & 0.084237 \\
3 & 0.09616 & 32 & 0.070004 & 61 & 0.101012 & 90 & 0.055611 & 119 & 0.126723 \\
4 & 0.077762 & 33 & 0.189997 & 62 & 0.118581 & 91 & 0.158906 & 120 & 0.096463 \\
5 & 0.120057 & 34 & 0.133908 & 63 & 0.073964 & 92 & 0.132694 & 121 & 0.140216 \\
6 & 0.028763 & 35 & 0.073813 & 64 & 0.0418 & 93 & 0.098446 & 122 & 0.055452 \\
7 & 0.118287 & 36 & 0.110664 & 65 & 0.31873 & 94 & 0.230228 & 123 & 0.077846 \\
8 & 0.094915 & 37 & 0.107742 & 66 & 0.152339 & 95 & 0.070645 & 124 & 0.071483 \\
9 & 0.0687 & 38 & 0.095386 & 67 & 0.088785 & 96 & 0.320208 & 125 & 0.063781 \\
10 & 0.111906 & 39 & 0.192636 & 68 & 0.158405 & 97 & 0.048276 & 126 & 0.073432 \\
\hline
\end{tabular}




\begin{tabular}{cccccccccc}
\hline 11 & 0.1152 & 40 & 0.089359 & 69 & 0.103055 & 98 & 0.067269 & 127 & 0.092611 \\
12 & 0.100854 & 41 & 0.111557 & 70 & 0.100654 & 99 & 0.094332 & 128 & 0.046648 \\
13 & 0.125419 & 42 & 0.090106 & 71 & 0.139613 & 100 & 0.041683 & 129 & 0.190098 \\
14 & 0.028025 & 43 & 0.092748 & 72 & 0.157462 & 101 & 0.072023 & 130 & 0.092356 \\
15 & 0.066386 & 44 & 0.133131 & 73 & 0.09616 & 102 & 0.070004 & 131 & 0.101012 \\
16 & 0.081724 & 45 & 0.078237 & 74 & 0.077762 & 103 & 0.189997 & 132 & 0.118581 \\
17 & 0.044307 & 46 & 0.059184 & 75 & 0.120057 & 104 & 0.133908 & 133 & 0.073964 \\
18 & 0.062308 & 47 & 0.062601 & 76 & 0.028763 & 105 & 0.073813 & 134 & 0.0418 \\
19 & 0.208625 & 48 & 0.084237 & 77 & 0.118287 & 106 & 0.110664 & 135 & 0.31873 \\
20 & 0.055611 & 49 & 0.126723 & 78 & 0.094915 & 107 & 0.107742 & 136 & 0.152339 \\
21 & 0.158906 & 50 & 0.096463 & 79 & 0.0687 & 108 & 0.095386 & 137 & 0.088785 \\
22 & 0.132694 & 51 & 0.140216 & 80 & 0.111906 & 109 & 0.192636 & 138 & 0.158405 \\
23 & 0.098446 & 52 & 0.055452 & 81 & 0.1152 & 110 & 0.089359 & 139 & 0.103055 \\
24 & 0.230228 & 53 & 0.077846 & 82 & 0.100854 & 111 & 0.111557 & 140 & 0.100654 \\
25 & 0.070645 & 54 & 0.071483 & 83 & 0.125419 & 112 & 0.090106 & 141 & 0.139613 \\
26 & 0.320208 & 55 & 0.063781 & 84 & 0.028025 & 113 & 0.092748 & - & - \\
27 & 0.048276 & 56 & 0.073432 & 85 & 0.066386 & 114 & 0.133131 & - & - \\
28 & 0.067269 & 57 & 0.092611 & 86 & 0.081724 & 115 & 0.078237 & - & - \\
29 & 0.094332 & 58 & 0.046648 & 87 & 0.044307 & 116 & 0.059184 & - & - \\
\hline
\end{tabular}

In this way, in addition to the performance values of the state of the presence of all the indicators, the number of problem indices, the values of the efficiency of the elimination modes will be obtained.

Now it's time to analyze the sensitivity of the results. At this stage, the goal is to determine the extent and effect of each of the factors on the performance of the unit in question (refinery gas). As described in methodology, for this purpose, the tensile average test is used between each single factor elimination mode with the presence of all factors. The results of the sensitivity analysis of the integrated model are presented in Table 9 .

Table 9. The sensitivity analysis results

\begin{tabular}{|c|c|c|c|c|c|}
\hline & Scenario & $\begin{array}{c}\text { Paired t-test } \\
\text { p-value }\end{array}$ & Mean & Result & $|D i f|$ \\
\hline \multirow{11}{*}{ Factors } & All factors & - & 0.10544 & - & - \\
\hline & Salary and wage & 0.001 & 0.18756 & $\mu_{F}<\mu_{I 1}$ & 0.08212 \\
\hline & Work environment & 0.004 & 0.17812 & $\mu_{F}<\mu_{I 1}$ & 0.07268 \\
\hline & Top management support & 0.106 & 0.09702 & $\mu_{F}=\mu_{I 1}$ & - \\
\hline & Awareness & 0.000 & 0.03691 & $\mu_{F}>\mu_{I 1}$ & 0.06853 \\
\hline & Reporting culture & 0.045 & 0.13965 & $\mu_{F}<\mu_{I 1}$ & 0.03421 \\
\hline & Teamwork & 0.102 & 0.10709 & $\mu_{F}=\mu_{I 1}$ & - \\
\hline & Preparedness & 0.011 & 0.05836 & $\mu_{F}>\mu_{I 1}$ & 0.04708 \\
\hline & Facility and welfare & 0.043 & 0.08767 & $\mu_{F}>\mu_{I 1}$ & 0.01777 \\
\hline & Training & 0.042 & 0.08762 & $\mu_{F}>\mu_{I 1}$ & 0.01782 \\
\hline & Job security & 0.017 & 0.06995 & $\mu_{F}>\mu_{I 1}$ & 0.03549 \\
\hline
\end{tabular}

As shown in Table 9, the average efficiency indicator for the presence of all factors is the same with the average performance indicator associated with eliminating senior management support and cooperative spirit and teamwork, and at 0.05 this test has not been rejected, Therefore, it can be concluded that the level of these factors is now at an acceptable level and has no significant positive or negative impact on the performance and unit performance. On the other hand, after eliminating some of the indicators, performance values have dropped significantly. This suggests the positive effect of the indexes on the performance and performance of the unit studied. For this purpose, the "payroll", "environmental conditions" and "reporting culture" indicators are the strengths of the system under review and are now at an appropriate level. In other words, the active staff at the refinery units of Fajr Jam is satisfied with the status of their salary and the working environment, and they evaluate it at the appropriate level. The results also indicate that the safety reporting culture is acceptable at the 
acceptable level. On the other hand, after the removal of some indicators, the performance values of the examined system have increased significantly. These indicators, which are considered to be system weaknesses and have a negative impact on system performance and performance, are: "awareness", "system planning and preparation for critical situations", "facilities", "education", and "Job security". The results show that FJG refinery employees are not aware of the safety issues and potential hazards as well as the details of safety incidents occurring in the system, and management also seeks to disseminate the full set of events and Safety issues are not. Also, employees are not satisfied with the existing amenities and they do not know enough. Due to the fact that most employees in the petrochemical and petrochemical industries are contracting, the results also indicate that employees are not satisfied with their job security. As we have seen, the results of the current study presented the performance evaluation of all indicators of job satisfaction and safety and industrial hygiene. Some indicators were normal, some at the negative level, and others were the strengths of the system and were on a positive level. Organizational management needs to plan and strengthen weaknesses and negatives to enhance employee satisfaction and performance as well as to increase preventive safety. Also, with long-term planning and a half-look at the strengths of their efforts to stabilize them.

\subsection{Prioritizing Indicators}

After analyzing the sensitivity and estimating the performance of each indicator, it is time to prioritize positive and negative indicators. Prioritization is based on the calculated weight for each of the indicators. In order to calculate the weight of each indicator, the difference between the mean values of efficiency before and after the removal of the index is calculated. After estimating this value for all indices, the values must be normalized and finally weights are reported in percentages.

\section{Verification and Validation}

Due to the fact that artificial neural networks are data-driven and always encountered with some kind of error, the results and findings of the experiment should be verified and evaluated. Although, artificial neural networks with data-driven nature and considering linear and nonlinear relationships in data are a dominant and superior method for assessing the boundaries of performance and performance. However, due to the existence of some errors in each network, and the potential for potential errors such as over-training, it is necessary to evaluate the results and findings of the research in a precise manner. Given the fact that data envelopment analysis is based on mathematical models, it provides accurate and reliable results. In this research, we use data-based algorithm-based algorithm to validate and validate the results of an artificial neural network algorithm.

The implementation of the algorithm based on data envelopment analysis is similar to the artificial neural network algorithm, except that in this method, data envelopment analysis techniques are used to compute performance values.

Now, in order to validate and validate the results of the algorithm based on neural networks by the results of data envelopment analysis, we need to examine the correlation coefficient between the performance values of the two approaches in the presence of all the indicators. Given that the two methods are essentially different in nature, for example, the neural network approach considers nonlinear relationships among variables, while the data envelopment analysis method uses a linear programming model to estimate the efficiency values. Therefore, there is no need for average performance values, but given the fact that both approaches are based on the estimation of the efficiency boundary, the process of data must be similar and verifying each other.

Therefore, we use the Pearson correlation test between the efficiency values obtained from both methods in the presence of all the indices. This test is done in Minibar software version 17. The result shows that the calculated Pearson Correlation Coefficient is 0.883 , which indicates the proper and acceptable performance of the network algorithm's neural network algorithm as compared with the data envelopment analysis algorithm.

\section{Conclusion}

Regarding the current research goal that performance evaluation of factors affecting the performance of FJJ staff was first, effective indicators were identified and the conceptual model of the problem was designed. After designing the conceptual model and the standard questionnaire, the required data were collected. Then, the validity and reliability of the collected data were evaluated using T-test and Cronbach's alpha. Then, using an algorithm based on the neural network, we calculated the efficiency index for the presence of all factors. Afterwards, we eliminated each factor in each repetition, and again we calculated the efficiency values. Then, using statistical methods such as t-test mean t, we evaluated and sensitized the obtained results. The results were as follows:

The average performance indicator for the presence of all factors with the average efficiency index is related to 
eliminating the factors of support of senior management and the spirit of cooperation and teamwork is the same and at the level of 0.05 this test is not rejected, it can be concluded that the amount of these factors is now at the level Are acceptable and have no significant positive or negative impact on the performance and performance of the unit. On the other hand, after eliminating some of the indicators, performance values have dropped significantly. This suggests the positive effect of the indexes on the performance and performance of the unit studied. For this purpose, the "payroll", "environmental conditions" and "reporting culture" indicators are the strengths of the system under review and are now at an appropriate level. In other words, the active staff at the refinery units of Fajr Jam is satisfied with the status of their salary and the working environment, and they evaluate it at the appropriate level. The results also indicate that the safety reporting culture is acceptable at the acceptable level. On the other hand, after the removal of some indicators, the performance values of the examined system have increased significantly. These indicators, which are considered to be system weaknesses and have a negative impact on system performance and performance, are: "awareness", "system planning and preparation for critical situations", "facilities", "education", and "Job security". The results show that FJG refinery employees are not aware of the safety issues and potential hazards as well as the details of safety incidents occurring in the system, and management also seeks to disseminate the full set of events and Safety issues are not. Also, employees are not satisfied with the existing amenities and they do not know enough. Due to the fact that most employees in the petrochemical and petrochemical industries are contracting, the results also indicate that employees are not satisfied with their job security. As we have seen, the results of the current study presented the performance evaluation of all indicators of job satisfaction and safety and industrial hygiene. Some indicators were normal, some at the negative level, and others were the strengths of the system and were on a positive level. Organizational management needs to plan and strengthen weaknesses and negatives to enhance employee satisfaction and performance as well as to increase preventive safety. Also, with long-term planning and a half-look at the strengths of their efforts to stabilize them.

According to the results obtained from the sensitivity analysis of the organization's management, it should first consider the top priority negative indicators and prioritize them, as their promotion has the greatest impact on the performance and safety of the organization. To this end, priority is given to the negative indicators of "awareness", "system readiness for critical situations" and "occupational safety". Also, the results and findings indicate strong points in the organization, which require side-by-side programs to maintain and consolidate them. Based on the results, the unit management can enhance the performance of human resources and make the system more precautionary safety in a more resistant and better condition.

\section{Acknowledgments}

This research was supported by the Natural Science Foundation of Shanghai (15ZR1401600)

\section{References}

Azadeh, A., Haghighi, S., Motevali, A. S. M., \& Saedi, H. (2013a). A new approach for layout optimization in maintenance workshops with safety factors: The case of a gas transmission unit. Journal of Loss Prevention in the Process Industries, 26(6), 1457-1465. https://doi.org/10.1016/j.jlp.2013.09.014

Azadeh, A., Haghighi, S., Motevali, Z. M., \& Khaefi, S. (2015a). Performance evaluation of Iranian electricity distribution units by using stochastic data envelopment analysis. International Journal of Electrical Power \& Energy Systems, 73, 919-931. https://doi.org/10.1016/j.ijepes.2015.06.002

Azadeh, A., Roohani, A., \& Haghighi, S. M. (2015b). Performance optimization of gas refineries by ANN and DEA based on financial and operational factors. World Journal of Engineering, 12(2), 109-134. https://doi.org/10.1260/1708-5284.12.2.109

Azadeh, A., Saberi, M., Rouzbahman, M., \& Saberi, Z. (2013b). An intelligent algorithm for performance evaluation of job stress and HSE factors in petrochemical plants with noise and uncertainty. Journal of Loss Prevention in the Process Industries, 26(1), 140-152. https://doi.org/10.1016/j.jlp.2012.10.004

Azadeh, A., Yazdanparast, R., Zadeh, S. A., \& Zadeh, A. E. (2017). Performance optimization of integrated resilience engineering and lean production principles. Expert Systems with Applications, 84, 155-170. https://doi.org/10.1016/j.eswa.2017.05.012

Faed, A., Chang, E., Saberi, M., Hussain, O. K., \& Azadeh, A. (2015). Intelligent customer complaint handling utilising principal component and data envelopment analysis (PDA). Applied Soft Computing. https://doi.org/10.1016/j.asoc.2015.02.018

Hadden, D. D., Estes, J., Peirce, G., Clark, M., \& Gibson, R. A. (2007). Performance-based training assessment: Google Patents. 
Heidari, R., Tavakkoli-Moghaddam, R., Yazdanparast, R., \& Aliabadi, L. (2017). A fuzzy data envelopment analysis for the supply chain resilience assessment: An Iranian car manufacturer. Recent Applications of Data Envelopment Analysis, 978(1), 122.

Hoque, K. (2013). Human resource management in the hotel industry: Strategy, innovation and performance: Routledge.

Huselid, M. A, Jackson, S. E., \& Schuler, R. S. (1997). Technical and strategic human resources management effectiveness as determinants of firm performance. Academy of Management journal, 40(1), 171-188. https://doi.org/10.2307/257025

Jiang, K. F., Lepak, D. P., Han, K. j., Hong, Y., K. A., \& Winkler, A. L. (2012). Clarifying the construct of human resource systems: Relating human resource management to employee performance. Human resource management review, 22(2), 73-85. https://doi.org/10.1016/j.hrmr.2011.11.005

Kennerley, M., \& Neely, A. (2002). A framework of the factors affecting the evolution of performance measurement systems. International journal of operations \& production management, 22(11), 1222-1245. https://doi.org/10.1108/01443570210450293

Liu, J., Ding, F. Y., \& Lall, V. (2000). Using data envelopment analysis to compare suppliers for supplier selection and performance improvement. Supply Chain Management: An International Journal, 5(3), 143-150. https://doi.org/10.1108/13598540010338893

Luna, D. E., Gil-Garcia, J., Ramon, L. R., Luis, F., Sandoval-Almazan, R., \& Duarte-Valle, A. (2013). Improving the performance assessment of government web portals: A proposal using data envelopment analysis DEA. Information Polity, 18(2), 169-187. https://doi.org/10.3233/IP-130302

Meyer, J. P., Paunonen, S. V., Gellatly, I. R., Goffin, R. D., \& Jackson, D. N. (1989). Organizational commitment and job performance: It's the nature of the commitment that counts. Journal of applied Psychology, 74(1), 152. https://doi.org/10.1037/0021-9010.74.1.152

Shields, J., Brown, M., Kaine, S., Dolle-Samuel, C., North-Samardzic, A., McLean, P., . . Plimmer, G. (2015). Managing employee performance \& reward: Concepts, practices, strategies: Cambridge University Press. https://doi.org/10.1017/CBO9781139197120

Thanassoulis, E. (1993). A comparison of regression analysis and data envelopment analysis as alternative methods for performance assessments. Journal of the operational research society, 1129-1144. https://doi.org/10.1057/jors.1993.185

Thanassoulis, E., Boussofiane, A., \& Dyson, R. G. (1996). A comparison of data envelopment analysis and ratio analysis as tools for performance assessment. Omega, 24(3), 229-244. https://doi.org/10.1016/0305-0483(95)00060-7

Wang, Y. M., Luo, Y., \& Liang, L. (2009). Fuzzy data envelopment analysis based upon fuzzy arithmetic with an application to performance assessment of manufacturing enterprises. Expert Systems with Applications, 36(3), 5205-5211. https://doi.org/10.1016/j.eswa.2008.06.102 


\section{Appendix. Questionnaire}

Table A1. The questionnaire items

1. To what extent do you know the company's paid salary in proportion to your responsibilities and responsibilities? (10 is very high and 1 is very low)

2. How satisfied are you with your work environment, such as temperature and humidity? (10 is very high and 1 is very low)

3. How do you assess the support of the top management of the organization from the staff and human resources of the company? (10 is very high and 1 is very low)

4. To what extent are you aware of safety issues and impending risks? (10 is very high and 1 is very low)

5. Can you easily report issues and safety issues to your superiors? (10 is very high and 1 is very low)

6. How much do you rate the team spirit and cooperation among the company's employees? (10 is very high and 1 is very low)

7. To what extent are senior executives planning and preparing for critical situations and impending safety issues such as fire? (10 is very high and 1 is very low)

8. How do you evaluate the company's facilities such as catering, accommodation and nutrition? (10 is very high and 1 is very low)

9. How do you assess the role and function of the company in training and human resource preparation for the tasks and activities? Do the staff see the necessary training before starting to work in the system? (10 is very high and 1 is very low)

10. How do you assess the job security and continuity of your company? (10 is very high and 1 is very low)

\section{Copyrights}

Copyright for this article is retained by the author(s), with first publication rights granted to the journal.

This is an open-access article distributed under the terms and conditions of the Creative Commons Attribution license (http://creativecommons.org/licenses/by/4.0/). 